\title{
MULTIVERSE CROW CONSCIOUS CLUSTERING ROUTING AND COVERAGE OPTIMIZATION HEALTH MONITORING ALGORITHM DURING COVID-19 PANDEMIC
}

\author{
Cosmena Mahapatra ${ }^{1,2 *}$, Ashish Payal ${ }^{1 \dagger}$ and Meenu Chopra ${ }^{2 \ddagger}$ \\ ${ }^{1}$ University School of Information Communication and Technology, Guru Gobind Singh \\ Indraprastha University, Delhi, India \\ ${ }^{2}$ Information Technology, Vivekananda Institute of Professional Studies, Delhi, India
}

\begin{abstract}
Covid-19 crisis has put the healthcare system under stress due to the shortage of healthcare workers in hospitals, the hour of the need is a highly flexible but dependable as well as an system which can alert hospital authorities immediately through centralized relay about the status of a patient if he/she becomes critical. Such a system may be built using a Big Data enabled Wireless Sensor Networks, which may be set up to monitor patients inside a hospital which shall be monitored through live channel feed by hospital monitoring authorities. The data from the sensors, through big data monitoring and analytics layer, will give crucial and timely results which will surely save many patients lives. In order to implement such a robust system, there is a need to build constant coverage and optimized clustering and routing algorithms which would solve this vital problem. This paper presents a novel energyconscious clustering routing and coverage algorithm for both equal and unequal distribution in the wireless sensor system and is called as Multiverse Crow Conscious Clustering Routing and Coverage Optimization Algo- rithm. The algorithm has been able to successfully attain the equilibrium of Wireless Sensor Network community energy consumption, enhance energy efficiency, and extend lifespan of the entire network. From the coverage point of view, the algorithm has been able to attain the distribution optimization at a greater rate with a decrease cost, and increase the efficacy of this algorithm. To prove its effectiveness, we have compared the proposed algorithms to multiple Nature Inspired Optimized WSN scenarios.
\end{abstract}

Keywords: WSN, MVO, Crow Search, Hybrid Optimization, QoS, Coverage Area

\section{Introduction}

Constant positive research has led to the increase in efficiency of wireless sensor nodes which in turn is leading to the building of various critical wireless sensor networks (WSN) applications which require the random and dense deployment of batterypowered sensor nodes in a system which is often left un-monitored to their sensing functionality Rashid and Rehmani (2016); Zou (2020); Mukherjee and Das (2020). The issue of coverage area connectivity magnifies many would if the application is based on Directional WSN (DWSN) Jia et al. (2017). These WSN specialize in sending the video of the area being monitored thus scope in various applications such as smart city management, monitoring of highly vulnerable terrain or it may be used for monitoring industrial applications. These directional sensors are powered by lithium batteries having limited energy. This means that if the nodes' energy is prematurely used up, the Quality of service (QoS) of the new network is hit greatly. It must be emphasized here that having data coverage connectivity and preservation would be the most indispensable function to ensure QoS in WSN in addition to the need for ensuring low energy intake. Thus, there arises a need for creating a balance the need for optimum coverage by reducing blind coverage areas and coverage area overlapping, but also to prolong the life of wireless sensor networks by using optimum Cluster formulation and data routing techniques. It has been noticed by various researchers that DWSN sensors have a huge redundancy ratio due to its rotating nature of the sensory direction. Redundancy ratio is directly proportional to the area covered by the coverage 
algorithm; this means that as the coverage area of WSN increases we can see a proportionate increase in its redundancy ratio. It is thus imperative that coverage area optimization of WSN must include this as a critical criterion in its algorithm.

As to the energy consumption aspect of a WSN optimization problem, there are two main areas where the researches may focus. The first is increasing the energy efficiency during the process of sensing, where sensors scan the area for available nodes. The other is to increase the energy efficiency during the relay of data between the sensor nodes by decreasing the en- ergy consumption during this process. These two areas may be effectively tackled through an optimized clustering and routing technique that optimizes the WSN by decreasing the energy expenditure in both the identified problem areas. Furthermore, in most WSN's it has been found that sensors spatially close to each other, often report redundant data to their base stations. In such a scenario also, clustering algorithms are implemented which create clusters of sensor nodes geographically close to each other. Also, to have an optimized solution for WSN, the research must focus on a sensor node that has primary function to be an active sensor node and transmit captured or sensed data and it would take its secondary role of a detector when it is off duty or enters power-save mode.

Another aspect to be noted is that Evolutionary or Nature Inspired algorithms have been identified to have inherent properties to optimize problem-solving. They generally focus on finding optimum solutions from the problem space. These optimum solutions may fall under the category of having local optima but it is preferable to have global optimum solutions in most cases. Nature has always provided its inhabitants the best globally applicable solution to any problem they may have come across. Researchers of Econometric and Mathematics found that the best solution to computer science optimization problem too was hidden within nature's complex ecosystem. Multiverse optimization algorithm (MVO) Mirjalili et al. (2016) is one such algorithm, it is an exploratory algorithm that is focused on finding the local as well as the global optimum solution through investigation of the search space and then utilization of the solutions identified through it. Another excellent algorithm to be studied is Crow Search optimization (CRSA) Askarzadeh (2016) algorithm which gives us globally as well as locally optimized solutions, thus making it a very potent algorithm.

After a thorough study of all aspects of wireless sensor networks and nature-inspired algo- rithm, in this work we have proposed a multi-objective algorithm under the title of Multiverse Crow Conscious Clustering Routing and Coverage Optimization Algorithm
(MVCCRO). To the best of our knowledge, this is the first time when a hybrid approach involving Multiverse and crow search algorithm has been proposed. This work aims to resolve the various identified work areas of WSN optimization which are listed below:

- MVCCRO will enable the WSN to successfully preserve coverage area as against the redundancy ration of directional WSN.

- Also, it enables WSN to have maximum connectivity at least energy consumption and at the same time, they maintain balanced energy consumption throughout the clusters.

- Furthermore, MVCCRO enables WSN to have an energy-efficient route for data packets from the originating $\mathrm{CM}$ to the base station. Unlike other clustering methods which utilize Euclidean distance, and

- Last but not the least MVCCRO makes use of stochastic Nature Inspired algorithm to achieve its goal of increasing energy efficiency; through this the WSN clusters can choosean optimum $\mathrm{CH}$, thereby reducing energy consumed during data transfer, minimum delay and maximum network lifetime.

The results of the study have confirmed that MVCCRO is able to increase the coverage ratio extensively at the same time maintaining an optimized risk-free status. We have compared the results with Grey Wolf Cuckoo Search (GWCSA)\$ Zhao et al. (2020) in our study to bring out the effectiveness of MVCCRO. We have organized the remaining sections in the following way, Section 2 of our paper, focuses on a brief review of literature that we have done to build our case. Section 2.1 reviews the Multiverse optimization and Crow search techniques respectively. We present our proposed solution in section 3. In section 4 we have suggested an MVCCRO based WSN application. Finally, Section 5 we have utilized to discuss our results and simulations in detail. The paper concludes with section 6 where we have summarized our paper.

\section{Review}

Several phenomena and processes in the nature are observed by the scientists and these processes are being adapted and replicated to the artificial systems. Nature has some well-organized and efficient 
ways to solve various real-world problems. Nature Inspired algorithms resembles the processes that are inspired from the nature. For example, the design of wings of an aircraft is inspired from the wings of an eagle(bird). Developing the optimum aerofoils in the wings of the aeroplane are inspired from the nature. Like wise the design of wind turbine, bionic car, bullet train are inspired from various components in the nature. The computational intelligence and nature inspired algorithms intersect with each other. The scientific reasons behind the phe- nomenon of nature are emerging as the solutions for the various real-world problems. The scien- tists have been trying to understand the nature by using several techniques and tools. The nature inspired algorithms has computation techniques such as problem-solving algorithms, synthesis of patterns, hardware, software problem solving techniques etc. Though various techniques are implemented to find out the answers for various real-world issues, nature inspired algorithms gained popularity due to its efficient way of finding the optimized solutions for the real-world problems. The techniques focus on the biology-based, chemistry-based and physics-based al- gorithms. The techniques and algorithms that are a part in the nature inspired techniques solve issues and give the optimized solution within reasonable time. The nature inspired techniques and algorithms should be implemented when the problem involves large number of variables or when the problem statement involves complex patterns or when the solution of the problem is not found using other traditional techniques. It is found that the swarm intelligence technique/algorithm is used as the most efficient algorithm to find the optimized solution of the problem. Optimization is an action of making something best and most effective with an using the resource or situation. In the process of optimization, the comparison of different methods or alternatives is done and the best out of it is chosen. Nature itself acts as an optimizer. For example, minimizing the drag is inspired from the birds, maintaining the lift at higher angles of attack is inspired from the humpback whale, maximizing the rigidity and minimizing the drag is inspired from boxfish, minimizing the micro pressure waves is inspired from kingfisher. There are different techniques for different types of problems. Nature inspired algorithms/techniques are divided into two methods. They are Evolutionary algorithmic techniques and swarm opti- mization algorithms. Swarm intelligence is technique of optimization that is population based. The techniques in swarm intelligence is inspired from the processes of nature like fish schooling and bird flocking. It is having few similarities with that of evolutionary algorithm techniques like genetic algorithms. The only difference is that swarm optimization techniques does not have recombination and mutation processes. These techniques have been useful to solve many problems of WSN, such as in one study the authors have suggested a very powerful clustering protocol that enables $\mathrm{CH}$ to communicate with CMs through optimum count. This algorithm simulation showed vast success with the proposed "Intersecting Sensor range Cluster Based Routing Algorithm" as compared to the prevailing electricity conserving methods in WSN G.N and C.D (2019). This study has introduced EEUCR which splits the cluster rings having un- equal dimensions into several smaller clusters. However, it allows rings that are closer to a base station, to have bigger circumference as compared to cluster rings which are far off. By this, it aims to balance the sensor node energy consumption. The algorithm though uses static cluster- ing yet $\mathrm{CH}$ is re-chosen, every time the nodes' energy goes down. The results of the algorithm show significant improvement in energy consumption as compared to other protocols, this in- creasing lifetime and establishing an energy balance among sensor nodes Handa et al. (2019)

In this paper, authors have proposed SSMOECHS Lee et al. (2019) which they have simu- lated and have been able to show enhancement in the network lifetime by $13.4 \%$, $7.1 \%, 34.6 \%$, and $1.8 \%$ respectively. The algorithm works by selecting $\mathrm{CH}$ from sampling CMs population. The sampling is done through SSMOECHS. The algorithm describes ways to pick up $\mathrm{CHs}$

through the discussed process. The authors of this study have proposed the EECRP-HQSND- ICRM algorithm of WSN. The algorithm is based on data redundancy removal. The algorithm does this by splitting larger areas into small 4 clusters, with each cluster having its own $\mathrm{CH}$ and $\mathrm{CMs}$. It makes use of the Dijkstra s algorithm to route the data through optimized transmission paths. The algorithm has been strong on network lifetime Ullah et al. (2020). Authors in this paper have successfully simulated a distinct result-based clustering approach for all types of clustering which has proved successful in efficiently managing energy efficiency Tripathi et al. (2018). This study has reviewed the major challenges and difficulties faced by coverage and connectivity algorithms. They have raised the pertinent issue of a new algorithm to solve the said WSN challenges Arjunan and Pothula (2019). A brief analysis of different "unequal clus- tering strategies" with their aims, attributes, etc. is introduced in this study. Additionally, the paper also builds several different sensor node properties, Cluster Head (CH) properties, and clustering procedure Sundaran et al. (2017).

\section{Whale}

Clustering 
Optimization algorithm is another nature inspired algorithm which when compared with PSO, ABC, GA, DE and K-Means has shown greater optimization Nasiri and Khiyabani (2018). Swarm optimization has different particles which are the primary sources for finding the optimized solution. This technique follows the behavior of bird flocking. The group of birds travel together and search for the food. The birds do not know the location of the food but with each iteration the birds will have some idea about the distance that has to be traveled. The bird following the good strategies to find the food and the bird that is nearest to the food finds the food. Likewise, the same bird flocking strategy is implemented in the particle swam optimization technique. Here the particles act as the birds finding for the effective and optimized solution. In this technique each particle is assigned with the fitness value. This fitness value decides the velocity of the particle that fly through the space of the problem following the current optimized particles. The optimized particles are iterated repeatedly and two best solutions are chosen Al-Madi et al. (2019).

An interesting study shows how hybrid optimization algorithm may combine MVO and FNN together to detect data failure during aggregation in WSN Dao et al. (2020). The study proposes to provide an alternate resource to intuit data failure and formulate an alternate effective way to forward WSN related crucial data to BS from $\mathrm{CH}$.

Multiverse optimization algorithm is based on Multiverse wormhole whole theory of physics. As per the authors, this theory promotes the existence of multiple universes through multiple

big bangs. In short, Multiversity is the opposite of the universe as it states the existence of not one but multiple universes Morris and Thorne (1988). These universes may intermix with each other by either socializing (positive) or colliding (negative) function. Furthermore, there exist different physical manifestations of multi-verse namely white hole, black hole, and wormholes. White holes result in a Big bang which is triggered by universes colliding with each other. How- ever, not much can be found on wormholes, which essentially remain as fiction but the theory which has been proven is the existence of black holes, due to which the other two manifestations also hold ground.

Black holes as discussed before, have been proven to exist in the universe, and through them, assumptions have been made to the existence of white holes. Black holes are massive gravitational masses that draw and capture even light inside them and bounce it outside at almost twice the speed of light at different time intervals, thus making the phenomena almost impossible to catch. On the other hand, wormholes are conceptual portals to another universe. Multi- verse theory further theorizes that the universes are ever-growing in nature, and their stability holds great significance in order to form planets, suns, and stars. The most important feature of the multi-verse theory lies in the fact that the cyclic nature of multi-verse exists only because of the interaction of white, black, and worm holes thus forming the basis of MVO. MVO algorithm is thus a populace-based nature inspired algorithm that splits the search space in two steps namely investigation and utilization. MVO optimization has the following assumptions:

1. The greater is the speed with which the universes expand; greater is the chance of a white hole.

2. The chance of having a black hole diminishes in a universe if the expansion speed of the universe is very fast.

3. White holes exist in universes which have a greater expansion rate. Universes that have less expansion rate result in absorption and teleportation of objects into them through Black holes.

4. Wormholes enable transportation of objects between universes irrespective of the speed of expansion of the universes.

The above Figure 1 shows the concept of wormhole between universes. To mathematically simulate the white/black Pit tunnels and swap the items of universes, authors have used

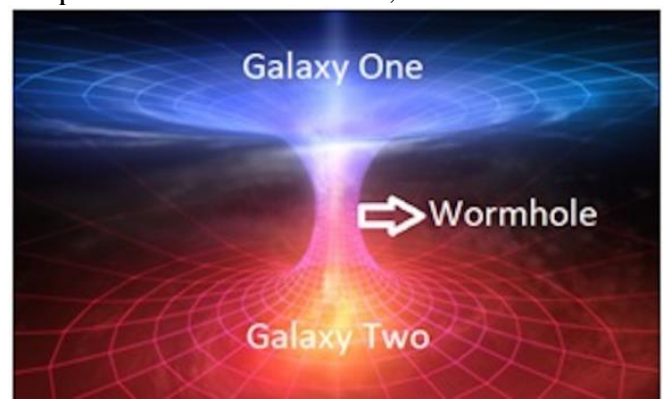

FIGURE 1: Illustration showing MVO wormhole theory

a Roulette Wheel mechanics. At each iteration, the algorithm forms the universes whose characteristics are predicated through universe growth (inflation) speeds and from these set of universes roulette wheel chooses a universe having a white hole and $\mathrm{N}$ is the Number of universes (candidate solutions):

$$
Y_{i}^{j}=\begin{array}{ll}
Y_{k}^{j} & r 1<N 1\left(U_{j}\right) \\
Y_{i}^{k} & r 2<N 1\left(U_{I}\right)
\end{array}
$$


In equation $1, Y^{j}$ indicates the parameters of both universes and, represents the universes

whose inflation (Normalized) is shown by N1 and takes random values between $[0,1]$.

Another study namely Crow search nature inspired algorithm (CRSA) till now has been used successfully in various applications such as image segmentation Fred et al. (2019), feature selection sayed et al. (2019), conduit size Abdelaziz and Fathy (2017) and AVR Bhullar et al. (2020) among other and has been proven to give optimized global outputs. The algorithm focuses on the food concealing nature of the crows. Crows are regarded as intelligent birds, which has good analytical skills as compared to other birds/animals, and can communicate to its flock, messages about dangers, and food sources. The algorithm works on 4 principal characteristics of crows:

1. They live in flocks, almost like humans.

2. They have memories that they can use for recalling places of food sources.

3. There flock has a hierarchy and hence followers will follow the leader.

4. They combine to protect their food sources from other birds/animals.

In the field of computational intelligence, naturebased algorithms have been emerging as the large and expanding stream. The different algorithms are used to find the best and optimized solution for every real-world problem. The algorithms are developed by the inspirations from nature and have been proven to be beneficial for WSN and related fields.

\section{Methodology}

In WSN, A node is elected as a cluster head $(\mathrm{CH})$ and all other clusters become the member of that cluster (CM). It is the responsibility of $\mathrm{CH}$ to balance data load by using various data aggregation methodologies. Nodes in a random WSN often do not give optimum network coverage; many times, this may result in a read being covered by fewer of nodes as compared to other areas making such areas critical to the functioning of a WSN. Also, to be noted is the fact that a $\mathrm{CH}$ uses more energy as compared to CMs. This means that if a coverage critical node is made a $\mathrm{CH}$, its expiry would prove critical to the whole WSN cluster. The other important criterion of focus here is connectivity. Connectivity is ensured by enabling a communicating WSN node to utilize intermediate sensors nodes for relaying information to base stations whenever needed. To have a robust and dependable WSN, connectivity is of prime importance.

For having good connectivity in a WSN, multiple sensors in a densely populated WSN may cover a large sub-area or it is to be utilized to activate and deactivate other sensor nodes; both of which contribute to increased WSN lifetime. Also, to be noted is data created by the sen- sors grow at a very high rate often leading to exponential growth. Traditional systems of data processing for information storage, processing, and reporting of big data WSN (for example highspeed computers and back end services) are overly costly to deal with. Furthermore, they can't deal with all the processing demands which could be needed for real-time procedures. Additionally, the majority of the events tracked at regular periods are mostly redundant or are small variations leading to a massive waste of information storage tools and communicating energy in relay and sensor nodes. This suggests that a lot of the data are of no attention, meaningless, and redundant. Therefore, unlike the case of average WSNs, in a Big Data-enabled WSN it's vital to collect and transmit a lot of information while reducing information latency in WSN-based large information systems. What's more, it's required to effectively eliminate redundancy of data and increase the energy efficiency vastly. The overlap between large data systems and WSNs is in the usage of data processing methods. For those WSNs facet, it might save their restricted resources. At precisely the same time, getting uncontaminated, redundancy free, and pertinent information would lower the excess data quantity in the face of the huge data system. Thus, it would decrease overload by detecting values from these sensor data quickly and effectively.

The proposed algorithm was framed in keeping in view the above highlighted needs of WSN. Broadly the proposed algorithm would first be framed by combining the two methods namely MVO and Crow Search togather by replacing a small part of MVO with crow search.

\section{The Proposed Algorithm}

As we know coverage problem is among those basic problems in WSNs since it has an immediate effect on the sensor's energy consumption and also the system lifetime Fanian and Rafsanjani (2019). The problem of area coverage can normally refer to the way to track the network area efficiently. There are lots of methods to catalog the coverage issues in WSNs. Depending upon the frequency of usage of the area being 
tracked, we may pigeonhole these coverage issues into continuous coverage issues or sweep coverage issues. Furthermore, cate- gorization of the area coverage issues may be done as per the type pf issue we wish to identify such as do we wish to identify area coverage, or are we more focused that means point coverage, or are we wishing to focus our self's on barrier coverage. Multiple type researches have been done to identify area coverage issues on the basis of levels from 1 to $\mathrm{k}$ (1 Level $-\mathrm{K}$ levels). Coverage problems can never be fully solved until connectivity within the area to be covered is given importance, which means we need to focus also on the type of connectivity needed within a WSN i e. connectivity-conscious coverage protocols or even non-connectivity aware coverage protocols.

What's more, coverage protocols could be categorized based on the adopted algorithm traits, into either dispersed protocols or centered protocols. Centralized coverage protocols could be categorized into an evolutionary algorithm (EA) Ma et al. (2018) based protocols or non-EA established protocols. Additionally, coverage protocols could be categorized based on the system version of this system. There are four features below the machine design: detector location awareness (conscious or unaware), detector freedom models (stationary, cell phone or ahybrid of both), detector installation models (deterministic or random), and detector sensing version. Sensing versions are widely categorized, depending on the sensing capability, into two different types: deterministic sensing models and probabilistic sensing versions. Sensing models may also be categorized, depending on the management of the sensing array, into directional sensing versions or Omni-directional sensing versions.

Optimization of coverage protocols is necessary if one wishes to make an efficient coverage protocol. Hence, to do this, we must make an informed choice of do we wish to make our pro- tocol coverage aware or do we should it run with sleep scheduling protocols. In the first case, coverage optimization occurs before the installation of the phase whereas in the second case occurs after the installation. Network topology-based classification for sleep scheduling pro- tocols may be grouped into a cluster-based or tree-based sleep scheduling protocols. Keeping the above in mind we have combined both the beneficial algorithms into one to create a hybrid form of Multiverse Crow Search Optimization algorithm, which has proved to effectively and accurately help in generating globally acceptable solutions. The algorithm has been found to give better coverage as covered to the other algorithm. MVCCRO algorithm is divided into 4 parts, the details of each part are as follows:

\section{Coverage Verification}

This part of the algorithm is used to optimize the sensor node's coverage area through verifica- tion. The steps of the algorithm are as follows:

1. Number of sensor nodes: $s=\{s 1, s 2, \ldots, s n\}$

2. The length and width of the target area: $1, b$

3. Calculate the coordinates of sensor nodes Asl, Bsh, Asl, Bsh

4. for $i=1$ to $n$ do

5. for $\mathrm{A}=$ Asl to Ash do

6. for $\mathrm{B}=\mathrm{Bsl}$ to Bsh do

7. if (the original point $\mathrm{P}$ is $\mathrm{S}(\mathrm{A}, \mathrm{B})$ ),

8. then check if $\mathrm{C}[\mathrm{A}][\mathrm{B}]=1$

9. end if

10. if (new point $P$ is being covered by 2 sensor nodes)

11. then justify $R[A][B]=1$

12. end if

13. End all for loops

\section{Cluster Head Selection}

The next task of the algorithm has a task to select an optimum $\mathrm{CH}$ to reduce the energy expendi- ture incurred during data transmission (received from sensor nodes, transmitted to sensors and base station).

Number of sensor nodes: $s=\{s 1, s 2, \ldots, s n\}$

1. $\mathrm{CH}$ Group $=\left\{\mathrm{CH}_{1}, \mathrm{CH}_{2}, \ldots, \mathrm{CH}_{n}\right\}$ and $\mathrm{CH}$ (Ids for nodes which become members): i_CH

2. Residual Energy $\{$ Sensors $=$ SN_E $\}$ and $\left\{\mathrm{CH}=\mathrm{CH} \_\mathrm{E}\right\}$

3. for $\mathrm{i}=1$ to $\mathrm{n}$ do

4. if $\mathrm{SN} \_\mathrm{E}>0$ then

5. Calculate i_CH(I)=1 for each $\mathrm{CW}(\mathrm{Si}, \mathrm{CHi})$

6. For 
$\mathrm{j}=2$ to $\mathrm{k}$ do

7. Calculate $\mathrm{CW}(\mathrm{Si}, \mathrm{CHj}) \&$ if $\mathrm{CW}(\mathrm{Si}$, $\mathrm{CHj})>$ temp then temp $=\mathrm{CW}(\mathrm{Si}, \mathrm{CHj})$

8. i_CH(i) $=\mathrm{j}$

9. end all loops

\section{Optimization Clustering \& Routing}

The third part of the algorithm uses Mutliverse Optimizer to optimize the Clustering and Rout- ing within the WSN

1. Number of sensor nodes: $s=\{s 1, s 2, \ldots, s n\}$

2. Predefined MVO Universe size

3. Number of universes $=\mathrm{U}=\mathrm{n}$

4. Max. Iteration allowed $=$ mnum

5. Inertial Mass $=\mathrm{iM}$, Gravitational Force $($ Universal $)=\mathrm{G}(\mathrm{u})$, acceleration $=\mathrm{a}_{c c}$, Velocity $=\mathrm{V}_{t}$, Curr_Position $=\mathrm{C}_{P}$

6. Calculate Inflation rate of each universe that is considered for MVO (NI) and update best solution (bst_sol)

7. Initialize $\mathrm{iM}, \mathrm{G}(\mathrm{u}), \mathrm{a}_{c c}, \mathrm{~V}_{t}, \mathrm{C}_{P}$

8. for $\mathrm{i}=1$ to num do // Optimized Cluster Head

9. Calculate Fitness1(U1i), using Roulette Wheel Selection (NI)

10. Clustering (U1besti=U1i)

11. end for

12. for $\mathrm{t}=1$ to mnum do // Optimised Route Discovery

13. for $\mathrm{i}=1$ to $\mathrm{n}$ do

14. Update $\mathrm{V}_{t} \& \mathrm{C}_{P}$

15. Re-Calculate Fitness1(U1i), using Roulette Wheel Selection (NI)

16. Route_Discovery(U1besti=U1i)

17. end for

18. Output (CHO, OR) [optimized $\mathrm{CH}$, optimized route]

Copyright $(\subset$ Authors

\section{Establishing Optimum Coverage Area \& Redundancy Ratio}

The last part of the algorithm implements CRSA to optimize the Coverage Area and a balanced redundancy ratio for WSN

1. Number of sensor nodes: $s=\{\mathrm{s} 1, \mathrm{~s} 2, \ldots, \mathrm{sn}\}$

2. Set iteration to maximum iteration (Miter)

3. Randomly scatter $\mathrm{N}$ crows at $\mathrm{C}_{P}$

4. Initialize memory of each crow

5. for $\mathrm{i}=1$ to num do

6. while (iter $<$ Miter)

7. Pick random Crow and set the probability of awareness

8. Set value of $\mathrm{fl}$ ( $\mathrm{fl}$ is scaling factor, smaller fl gives global minima and larger values of fl gives global maxima

9. update the position and memory of each crow

10. ++Miter

11. end while

12. Calculate \& Output (OCR, RR) [Coverage Ratio, Redundancy Ratio]

13. end for

\section{WSN based Application for Covid - 19 Like Pandemics}

During Covid - 19, when the health infrastructure is already stressed a parameter of paramount importance is energy consumed. This is because due to this crisis, where the hospitals maximum bed capacity might have been $400-500$ beds and 100 ventilators at a given time, the new ca- pacity has been stretched to 1000 beds and 500-600 ventilators there by placing unprecedented energy requirement. The pandemic has definitely opened our eyes to the dangers of unknown viruses like it which may result in probably more devastating pandemics. It is time we started building a robust healthcare system, which addresses the shortage of healthcare workers in such situations. The advancemen 
ts in the AI field have left millions of people speechless, the ben- efits of this study are unprecedented. The ability of Artificial Intelligence to predict the rapid diffusion of virus and also the potential hot spots can play a vital role in tackling the virus at its initial stages. Also, early detection can help in containing the virus and thus, we can protect various other individuals from being affected. Considering the different technologies that we have at our disposal; it is observed that they are not able provide enough and faster assistance to tackle the virus. But, Artificial Intelligence can a big boon in this critical situation, as it has the ability to work with greater efficiency by accessing the data, using accurate algorithms.

\section{Selecting a store of abundant amounts of data}

An obvious advantage of using Artificial Intelligence for handling the Corona virus is its ability to store, manage abundant amounts of data. Also, complex data which are normally tough for databases or other technologies to handle can be easily handled by using Artificial Intelligence. Although, scientists have recommended that it is best to set certain rules for the Artificial Intelligence technology to work effectively in this scenario but there is nothing to worry whether rules will be obeyed as its one of the most promising features of Artificial Intelligence. The accurate and detailed analysis performed by AI can assist the doctors in using the most effective treat- ment plan and also, about the supply of resources such as medicines or other such necessities as required for treating the patient. Also, the supply chain of the health system can be handled by using this analysis done by AI can be helpful to send the requirements and form a better plan or design such as allocating new treatment centers or the ways to manage high population density areas. This can help in making a successful approach for ensuring that the resources reach each and every area or place or region that is in need of support for battling with this treacherous Corona Virus.

\section{Crucial role of Machine Learning}

Machine Learning is capable of collaborating abundant amounts of information with efficiency and repetitive process along with accurate, powerful algorithms which assist the software to understand and devour the process of identifying the patterns automatically without any man- ual work. Wide number of existing proofs regarding the service of machine learning helps to build up the trust and confidence regarding the performance of MI in tackling Corona Virus. Almost every health organization utilizes machine learning services at some or the other extent. Machine Learning is very simple like the natural language processing and the usage of this technology ranges from a minute scale to handling a vital system. Machine Learning can assist in allocating the medicines to the patients by using the diagnosis information and then, relating that information to the data regarding different kinds of diseases stored in the database. Also, the rapidness in which counter measures are being taken by the doctors and also the resource allotments to a specific area or hospital has the hand or assistance of Machine Leaning in some or the other way.

\section{Efficient Detection and Analysis}

It is believed that Machine Learning models soon will be able to read the CT and X-ray images for determining the range of pneumonia specifically. Also, these models will be capable enough to identify different kinds of pneumonia but then these models do require better information and scenarios to cross verify the data, understand the genetic data along with the previous scanned data of the patient before the pneumonia attack to compare and contrast to the present condition.

\section{Diagnosis}

Diagnosis considers all the required parameters such as the body temperature, heartbeat, respiration levels, and the blood oxygen content. Highly accurate devices implanted with the machine learning technology measure these parameters efficiently and accurately with non-invasive sensors. Also, the usage of machine learning is not just limited to the above-mentioned parameters as it can also measure and monitor other signs such as the motions, voice, weight, etc. The combination of different algorithms of Machine Learning can be utilized to predict the arrival or the disease progression so far in a victim and this information can be used to take preventive measures for protecting the patient. These devices or systems can help the doctors or victims take care of their health at home or in a closed space with less person-to-person interaction or assistance and thus, reducing the chances of diffusion of this disease to the doctors treating the patients or other assisting individuals.

\section{Artificial Intelligence and X-rays}

It is known that the Polymerase Chain Reaction is quite costly and also a highly time-consuming process. One of the standard procedures for detecting or identifying the COVID 19 are the chest $\mathrm{X}$-rays. But a simple or normal chest $\mathrm{X}$-ray is not sufficient to identify the virus or its extension into the lungs. Artificial Intelligence when collaborated 
with the chest $\mathrm{x}$-rays can assist in detecting the abnormal areas and thus, identifying the ground glass opacities in the lungs. This identification is very crucial to monitor or understand the COVID19 disease. This technique is highly beneficial for the patients placed in Intensive Care Unit (ICU).

\section{Tracing with the help of AI}

Popular technology giant companies such as Apple, Google have initiated the proposal of developing an android application for the purpose of tracing the COVID-19 victims. They have mentioned that this particular application would function with help of Bluetooth service. However, Indian government has ruled out this proposal by developing their very own Arogya Setu application. Also, Drones proved themselves to be helpful to the cause by assisting as monitoring devices for containment zones.
Keeping in view the various advantages of an AI based system, we propose a Nature Inspired system to further help all in fighting Covid 19. As illustrated in Figure 2, every patient admitted in these WSN enabled hospitals should be made to wear a Health monitoring WSN sensor band which would be relaying critical data to base stations. These Base stations would be connected to Big data cloud servers constantly, where analyzed results would trigger alarms for a centralized monitoring team, which would intern rush healthcare workers to identified critical patients. This system aims to save lives of Covid 19 patients as well as help in disease control of similar types, as patients affected by such diseases often unannounced turn critical thereby resulting in an untimely death. The proposed systems, welltimed alert would enable the hospital monitoring teams in dispatching health workers timely.

FIGURE 2: Architecture of Big Data-enabled WSN Patient Monitoring System
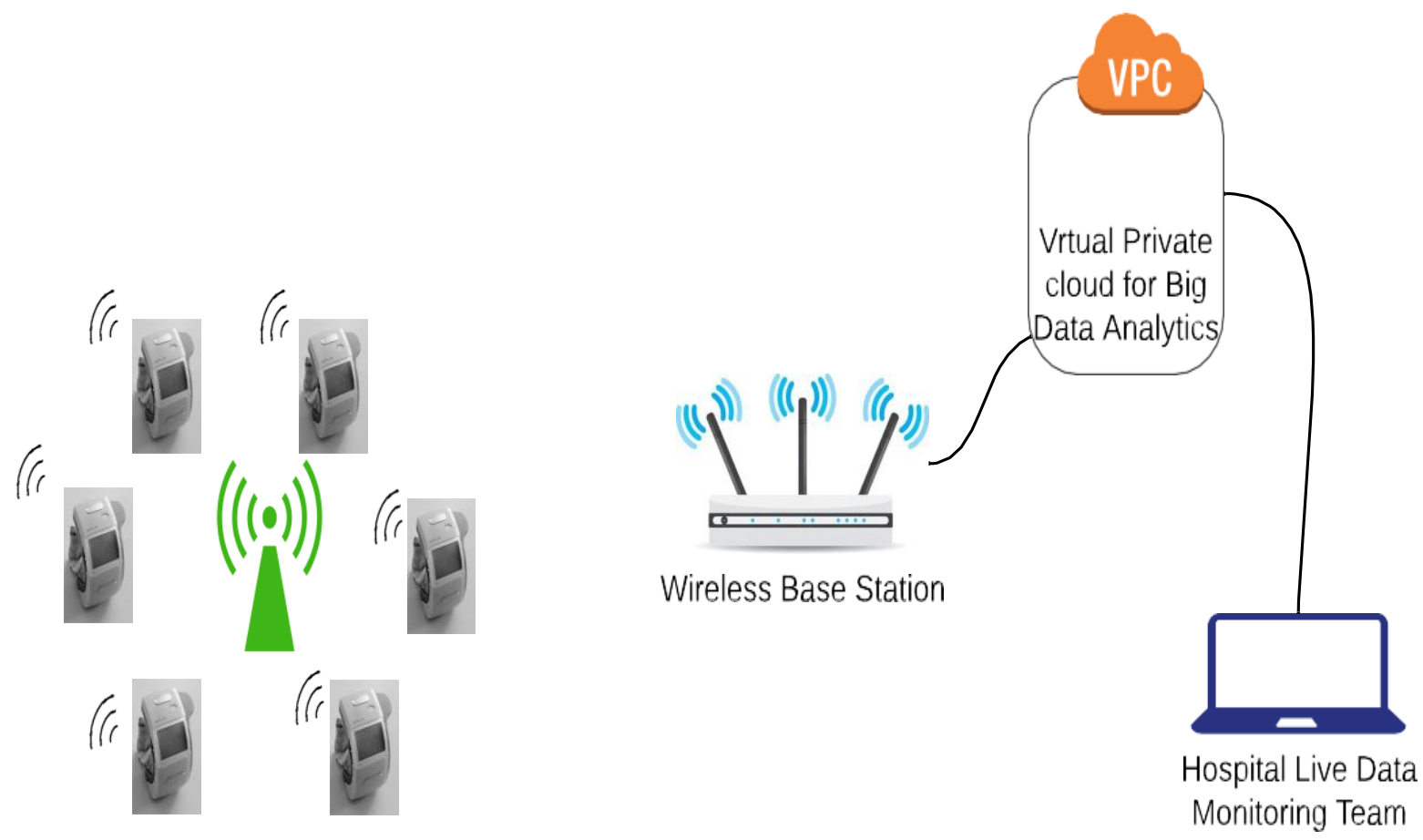


\section{Result and Simulation}

The simulation has been done in MATLAB 2019 QI (2012), parameters used for the simulation of MVCCRO and GWCSA are listed

as follows:

TABLE 1

Simulation Parameters for MVCCRO in Matlab 2019 environment

\begin{tabular}{|c|c|c|}
\hline S. No & Parameters Name & Parameter Value \\
\hline 1 & Target Area & $500 * 500$-meter square \\
\hline 2 & Base Stations & $(0,250)$ to $(250,250)$ \\
\hline 3 & No. of Sensors & 100 to 500 \\
\hline 4 & No. of $\mathrm{CH}$ & $6-60$ \\
\hline 5 & Sensing Range of Sensors & 60 meters \\
\hline 6 & Sensing Range of $\mathrm{CH}$ & 150 meters \\
\hline 7 & Initial energy of Sensors & 2 Joules \\
\hline 8 & Initial energy of $\mathrm{CH}$ & 10 Joules \\
\hline 9 & Packet size $\mathrm{P}$ & 4000 bits \\
\hline 10 & $\epsilon_{m}$ & $0.0013 \mathrm{e}-12$ \\
\hline 11 & $\epsilon_{f s}$ & $10 \mathrm{pJ} / \mathrm{bit} / \mathrm{m}^{2}$ \\
\hline 12 & $\mathrm{~d}_{0}$ & 86 meters \\
\hline
\end{tabular}

From the table, it is clear that the network coverage as also shown in Figure 3 and Figure 4 has seen effective growth when MVCCRO has been used as compared to GWCSA. Figure 3 summarizes the Routing and Coverage MVCCRO superiority over GWOCSA, the parameters chosen for comparisons are widely used in similar researches as discussed earlier.

The simulations assume that the sensor nodes in the wireless sensor area generate an equal amount of data packets in each round. We may assume that in a hospital at a particular time 500 is the count of Covid-19 patients. This may be changed as required. The maximum number of cluster heads is set to 60 . The area of Wireless Sensor Networks area is $500 * 500$. To be noted is that in the simulation we have is minimizing the maximum energy consumption Jacob and John (2012) of any cluster in one round as the fitness function.

The RMSE Yair and Altman (2014); Vinodha and Durairaj (2020) score in Figure 4 for MVCCRO is much less than established PSO Elhabyan et al. (2018) and GWCSO algorithms. Two other critical parameters of

MVCCRO performance measure is the Number of Dead 


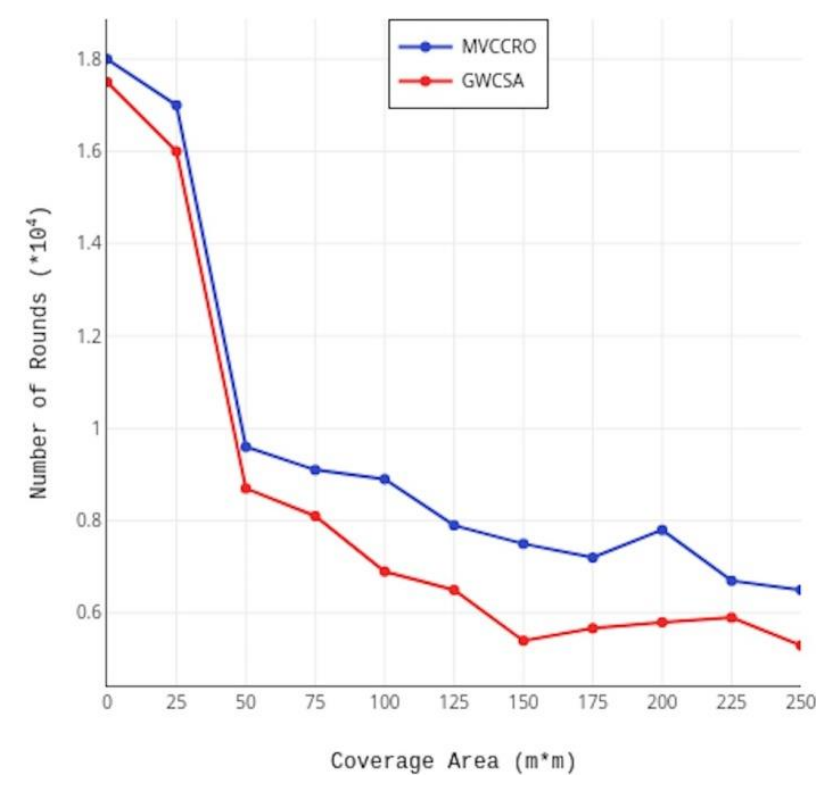

FIGURE 3: Network Coverage Graph for MVCCRO versus GWCSA

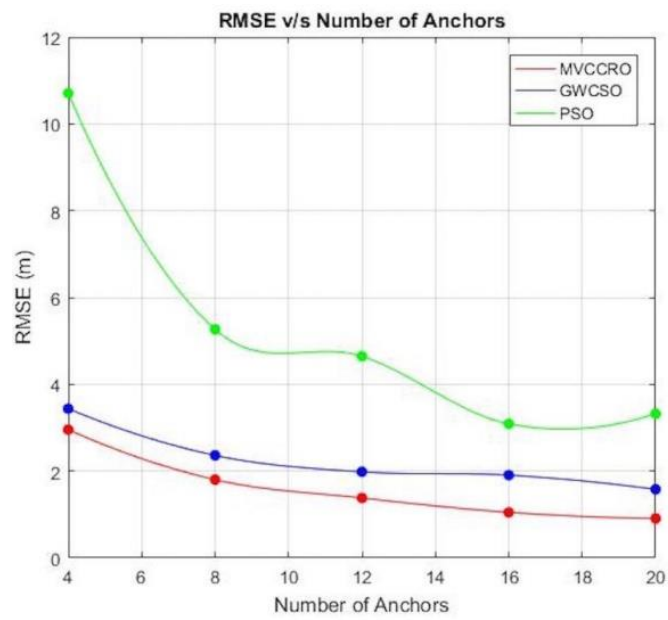

FIGURE 4: Comparative analysis of RMSE based performance

Sensor Nodes and Number of Dead Cluster Heads. As summarized, in Figure 5 and Figure 6 given below MVCCRO certainly outperforms forms GWCSA in both the parameters thereby increasing the network lifetime as the number of dead sensor nodes is effectively curtailed by MVCCRO as compared to GWCSA. Not only this, the number of dead cluster heads are also comparatively less in MVCRRO scenarios as compared to the network being managed by GWCSA.

As shown is Figure No. 7, MVCCRO algorithm is able to give us better packet delivery ratio 


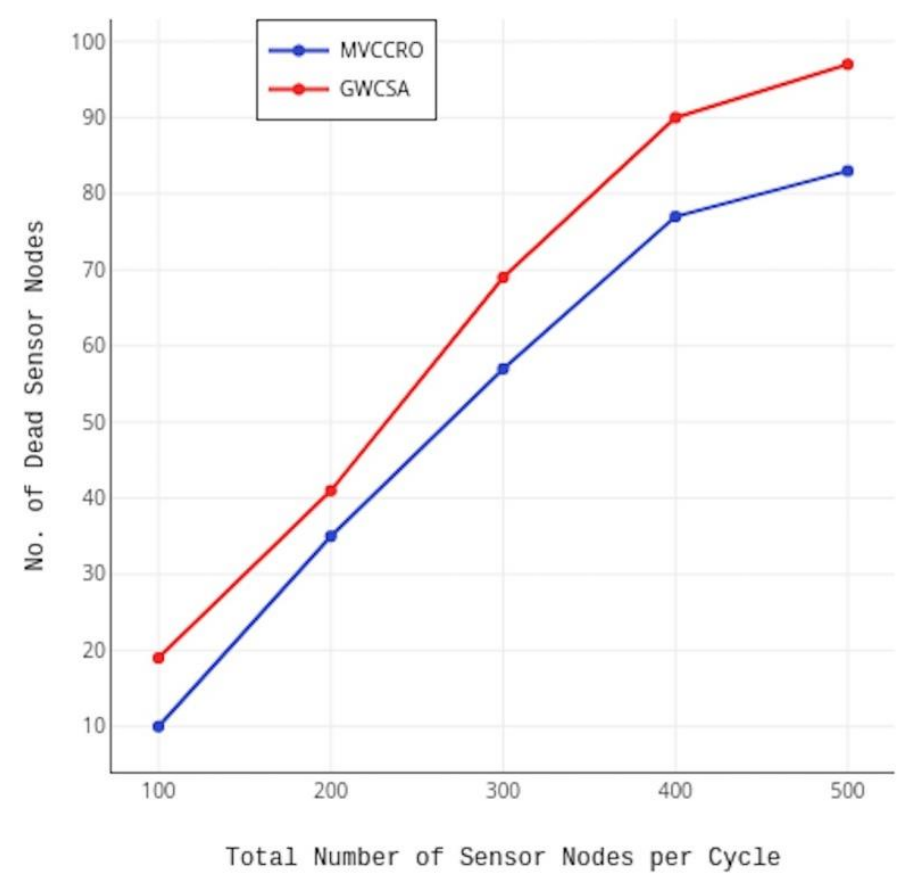

FIGURE 5: Network performance analysis on the basis of Dead Sensors nodes

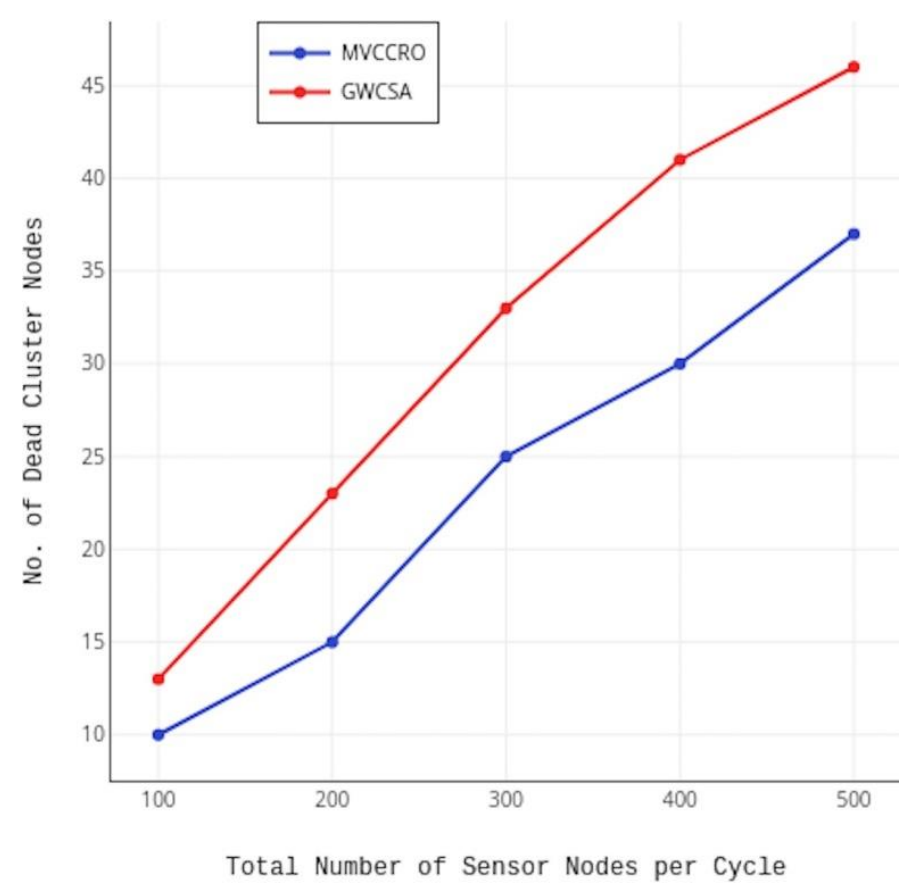

FIGURE 6: Performance analysis on the basis of dead CH's 


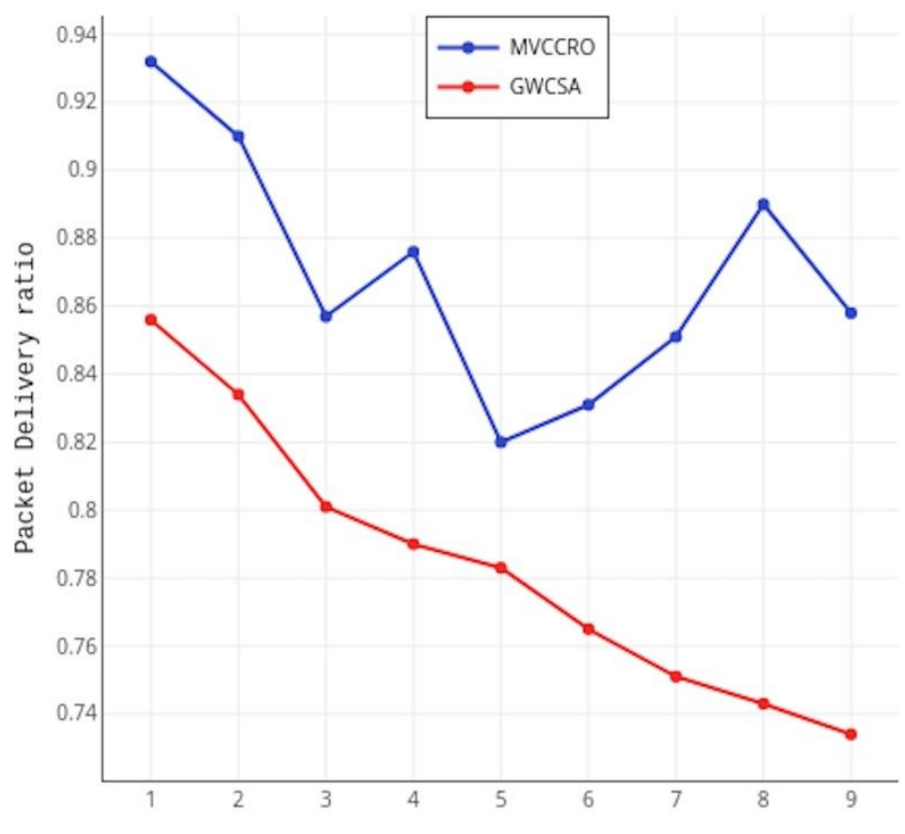

Max. Speed of Mobile Sensor Nodes (m/s)

FIGURE 7: Performance analysis of Networks Packet delivery ratio w.r.t Mobile Sensor nodes

as compared to GWCSA. Above Graph has been created using MATLAB \& PLOTLY Sievert (2020) to capture the dynamic nature of the mobile sensor node. The graph clearly depicts the downfall of GWCSA algorithm as the speed of mobile nodes increase, thereby having a bad effect on the packet delivery ratio.

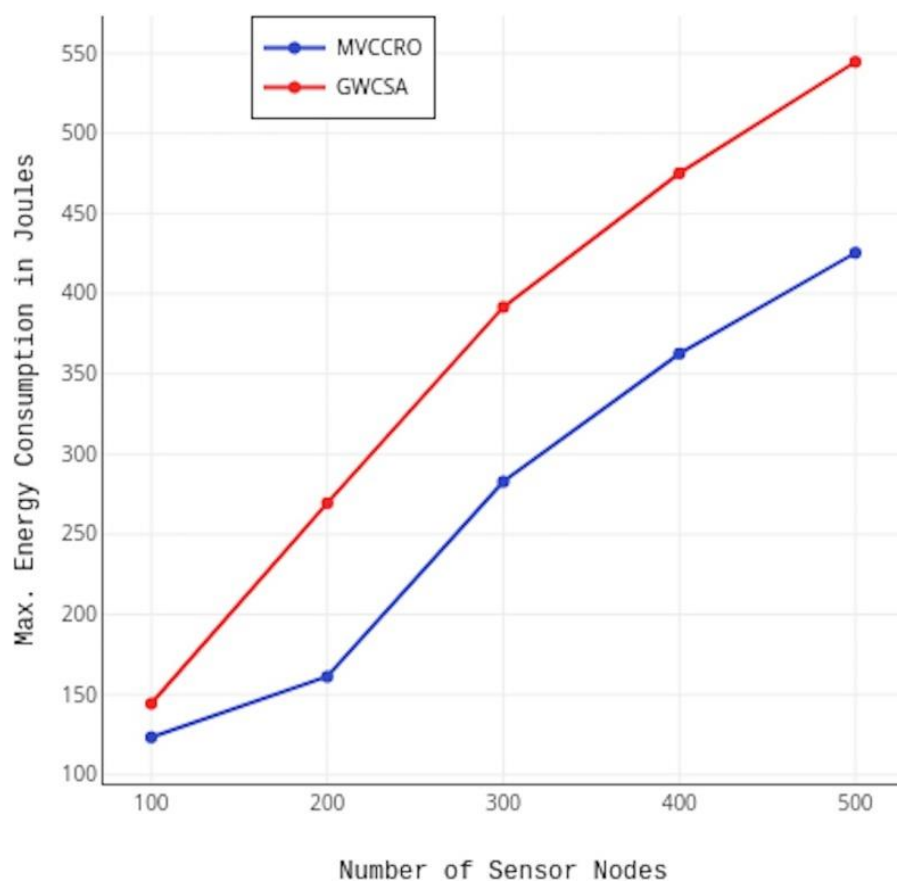

FIGURE 8: Total energy consumption comparison graph in Joules 
For such a Big Data Kim et al. (2019) enabled WSN to work successfully it is imperative that the total energy consumed by the networks must be kept less, so as to increase the network lifetime. The MVCCRO algorithm when compared to GWCSA, has shown very positive results in balancing energy consumed which is clearer in the Figure No 8:

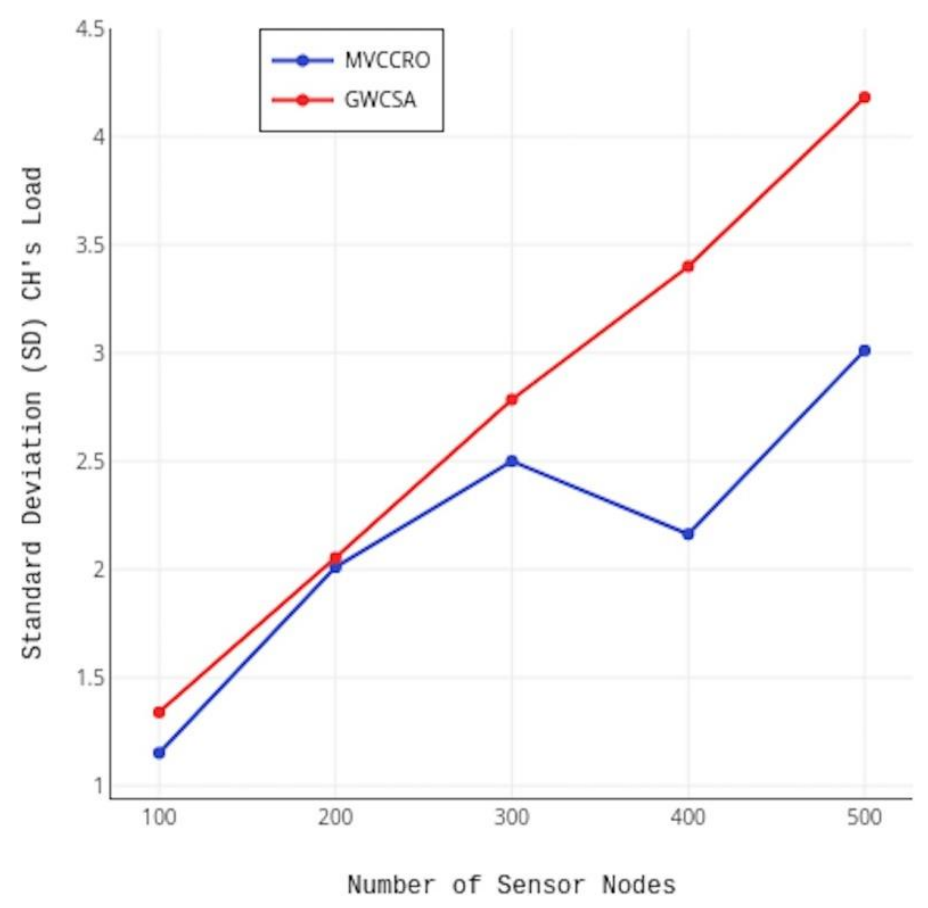

FIGURE 9: Change in Standard deviation of $\mathrm{CH}$ load with varying density of Sensors

The standard deviation of CHs' Load performance tests (Figure 9) indicate that the proposed system not only prolongs more efficiently the lifetime of cluster heads and audience members compared to other functions, but in addition, outperforms them into balancing the energy level of their cluster heads. As a result, the length of action of the cluster members in distinct clusters can also be balanced and protected.

TABLE 2

Average evaluation criteria for the algorithms

\begin{tabular}{llllll}
\hline AEC & PSO & GWO & MVO & CRS & MVCCRO \\
& & & & & \\
\hline Mean & $1.19 \mathrm{E}-05$ & $1.94 \mathrm{E}-05$ & $1.85 \mathrm{E}-06$ & $1.94 \mathrm{E}-05$ & $3.78 \mathrm{E}-06$ \\
Best & $1.07 \mathrm{E}-05$ & $8.32 \mathrm{E}-06$ & $3.15 \mathrm{E}-06$ & $2.32 \mathrm{E}-06$ & $2.19 \mathrm{E}-06$ \\
& & & & & \\
Worst & $1.29 \mathrm{E}-05$ & $3.24 \mathrm{E}-05$ & $2.24 \mathrm{E}-05$ & $3.24 \mathrm{E}-06$ & $5.10 \mathrm{E}-06$ \\
& & & & & \\
\hline
\end{tabular}


Table 2 summarizes the mean, best and worst evaluation criteria of all algorithms as men- tioned in the table; the best being MVCCRO. The results obtained show clearly that MVCCRO

is able to achieve minimum fitness function values and also giving optimal results.

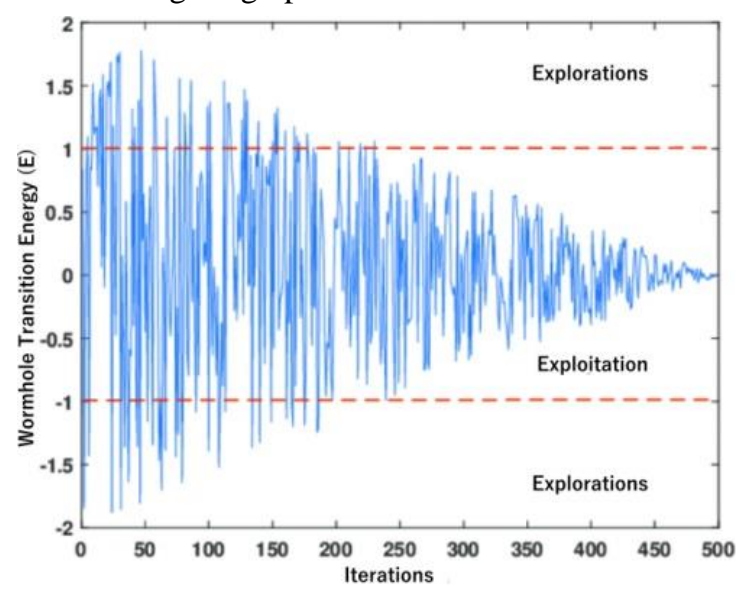

FIGURE 10: Effectiveness of MVCCRO through Exploration-Exploitation Phase Energy Map- ping

As shown in figure 10, the wormhole transition energy gradually diminishes as one moves from exploration to exploitation phases. This forms the basis for MVCCRO algorithm as it helps add randomization to our search space as the wormholes provide transitory path to optimum multiverse.

\section{Conclusion}

Covid 19 crisis has thrown many challenges for human existence. One of the sectors brutally hit by Covid 19 is health. Even developed countries like the USA, UK, Italy health facilities have been reeling under its immense pressure, with unprecedented demands on the healthcare workers and healthcare system itself. In such times it is only rational to have a Big data-enabled WSN to be set up for monitoring the patients continuously from a centralized point through a big data analytics platform and generate alarms for patients turning critical thereby providing them interventions timely. For this purpose, in this paper we analyzed and simulated in MAT- LAB, Multiverse Crow Conscious Clustering Routing, and Coverage Optimization Algorithm for mission-critical wireless sensor networks (MVCCRO). Primarily, the MVCCRO node sensing and cluster routing model when examined as well as analyzed has been found to have good network coverage, increased usage of nodes, and decreased energy consumption in-network coverage from the inertial weight procedure as compared to PSO, MVO, CRS and GWO opti- mized WSN.

\section{References}

1. Abdelaziz, A. Y. and Fathy, A. (2017). A novel approach based on crow search algorithm for optimal selection of conductor size in radial distribution networks.

2. Al-Madi, N., Faris, H., and Mirjalili, S. (2019). Binary multi-verse optimization algorithm for global optimization and discrete problems. International Journal of Machine Learning and Cybernetics, 10(12):3445-3465.

3. Arjunan, S. and Pothula, S. (2019). A survey on unequal clustering protocols in Wireless Sensor Networks. Journal of King Saud University - Computer and Information Sciences, 31(3):304-317.

4. Askarzadeh, A. (2016). A novel metaheuristic method for solving constrained engineering optimization problems: crow search algorithm. Computers \&amp; Structures, 169:1-12.

5. Bhullar, A. K., Kaur, R., and Sondhi, S. (2020). Enhanced crow search algorithm for AVR optimization. Soft Computing.

6. Daniel Francis Selvaraj, Balakrishnan S, Manopaul P, Coronavirus Disease (Covid19) Outbreak In India, International Journal of Pharmaceutical Research, Jan Mar 2021, Vol. 13, Issue 1, pp. 568-571. https://doi.org/10.31838/ijpr/202 1.13.01.086

7. Dao, T., Yu, J., Nguyen, T., and Ngo, T. (2020). A Hybrid Improved MVO and FNN for Iden- tifying Collected Data Failure in Cluster Heads in WSN. IEEE Access, 8:124311-124322.

8. Elhabyan, R., Shi, W., and St-Hilaire, M. (2018). Evolutionary-Based Coverage Control Mech- anism for Clustered Wireless Sensor Networks. In K., C., M., D. F., I., M., and B., S., editors, Wired/Wireless Internet Communications. WWIC, volume 10866. Springer.

9. Fanian, F. and Rafsanjani, M. K. (2019). Cluster-based routing protocols in wireless sensor networks: A survey based on methodology. Journal of Network and Computer Applications, 142:111-142.

10. Fred 
, L., Kumar, A., Padmanaban, S. N., Gulyas, P., B, Kumar, A., and H (2019). Fuzzy-Crow Search Optimization for Medical Image Segmentation. Applications of Hybrid Metaheuristic Algorithms for Image Processing, 890:171-188.

11. G.N, B. and C.D, D. J. (2019). Intersecting Sensor Range Cluster-based Routing Algorithm for Enhancing Energy in WSN. International Journal of Advanced Networking and Applications, 10(4):39383943.

12. handa, P., Panag, T. S., and Sohi, B. (2019). Reducing Delay of Wireless Sensor Networks Using Energy Efficient Unequal Clustering Routing Algorithm. International Journal of Sci- entific \& Technology Research, 8(11):3185-3189.

13. Jacob, J. M. and John, A. (2012). Improving Lifetime of Structured Deployed Wireless Sensor Network Using Sleepy Algorithm. In J., M., P., P., D.K., P., and A.J., K., editors, Eco-friendly Computing and Communication Systems. ICECCS 2012, volume 305. Springer.

14. Jia, J., Dong, C., He, X., Li, D., and Yu, Y. (2017). Sensor scheduling for target cover- age in directional sensor networks. International Journal of Distributed Sensor Networks, 13(6):155014771771364155014771771364 .

15. J.P. Ananth, S. Balakrishnan, S.P.Premnath, (2018). "Logo Based Pattern Matching Algorithm for Intrusion Detection System in Wireless Sensor Network", International Journal of Pure and Applied Mathematics, Volume 119, No. 12, 2018, pp. 753-762.

16. Kim, B.-S., Kim, K.-I., Shah, B., Chow, F., and Kim, K. H. (2019). Wireless sensor networks for big data systems. Sensors, 19(7):1565.

17. Lee, J. G., Chim, S., and Park, H. H. (2019). Energy-Efficient Cluster-Head Selection for Wireless Sensor Networks Using Sampling-Based Spider Monkey Optimization. Sensors, 19:5281-5281.

18. Ma, X., Li, X., Zhang, Q., Tang, K., Liang, Z., Xie, W., and Zhu, Z. (2018). A survey on cooperative co-evolutionary algorithms. IEEE Transactions on Evolutionary Computation, 23(3):421441.
19. M. Balasubramanian, S. Balakrishnan, Reflections on COVID-19 in India: A Survey, International Journal of Pharmaceutical Research, Oct - Dec 2020, Vol. 12, Issue 4, pp. 137-140. https://doi.org/10.31838/ijpr/2020.12.04.0 23.

20. Mirjalili, S., Mirjalili, S. M., and Hatamlou, A. (2016). Multi-Verse Optimizer: a nature- inspired algorithm for global optimization. Neural Computing and Applications, 27(2):495-513.

21. Morris, M. S. and Thorne, K. S. (1988). Wormholes in spacetime and their use for interstellar travel: A tool for teaching general relativity. American Journal of Physics, 56(5):395-412.

22. Mukherjee, P. and Das, A. (2020). NatureInspired Algorithms for Reliable, LowLatency Communication in Wireless Sensor Networks for Pervasive Healthcare Applications. In D., D., A., M., S., K. D., and N., D., editors, Springer Tracts in Nature-Inspired Computing. Springer.

23. Nasiri, J. and Khiyabani, F. M. (2018). A whale optimization algorithm (WOA) approach for clustering. Cogent Mathematics \&amp; Statistics, 5(1):1483565.

24. QI, A. (2012). Simulation framework of wireless sensor network (WSN) using matlab/simulink software.

25. Rashid, B. and Rehmani, M. H. (2016). Applications of wireless sensor networks for urban areas: A survey. Journal of Network and Computer Applications, 60:192-219.

26. Sayed, Ismail, G., Hassanien, Ella, A., Azar, and Taher, A. (2019). Feature selection via a novel chaotic crow search algorithm.

27. S. Balakrishnan, B. Persis Urbana Ivy and S. Sudhakar Ilango, "A Novel And Secured Intrusion Detection System For Wireless Sensor Networks Using Identity Based Online/Offline Signature", ARPN Journal of Engineering and Applied Sciences. November 2018, Vol. 13 No. 21, pp. 8544-8547.

28. S. Balakrishnan, Vinod K, B. Shaji. (2018). "Secured and Energy Efficient AODV Routing Protocol For Wireless Sensor Network", International Journal of Pure 
and Applied Mathematics, Vol. 119, No. 10c, 2018, pp. 563-570.

29. S. Balakrishnan, J.P.Ananth, L.Ramanathan, S.P.Premnath, (2018). "An Adaptive Energy Efficient Data Gathering In Wireless Sensor Networks", International Journal of Pure and Applied Mathematics, Volume 118 No. 21, 2018, pp. 2501-2510.

30. Sievert, C. (2020). Interactive Web-Based Data Visualization with $\mathrm{R}$, plotly, and shiny. CRC Press.

31. Sundaran, K., Ganapathy, V., and Sudhakara, P. (2017). Fuzzy logic based unequal clustering in wireless sensor network for minimizing energy consumption. In 2017 2nd International Conference on Computing and Communications Technologies (ICCCT), pages 304-309.

32. Tripathi, A., Gupta, H. P., Dutta, T., Mishra, R., Shukla, K. K., and Jit, S. (2018). Coverage and Connectivity in WSNs: A Survey, Research Issues and Challenges. IEEE Access, 6:2697126992.

33. Ullah, Z., Gagliardi, R., Gemikonakli, O., and Al-Turjman, F. (2020). Network Lifetime Effi- ciency Based on Equal and Unequal Size Clustering Strategies. In L., B., F., A., F., M., T., E., and M., T., editors, Web, Artificial Intelligence and Network Applications. WAINA 2020. Advances in Intelligent Systems and Computing, volume 1150 . Springer.

34. Vinodha, R. and Durairaj, S. (2020). Soft computing approach-based energy and correlation aware cooperative data collection for wireless sensor network. Journal of Ambient Intelli- gence and Humanized Computing. Yair, M. and Altman (2014).

35. Zhao, X., Ren, S., Quan, H., and Gao, Q. (2020). Routing Protocol for Heterogeneous Wireless Sensor Networks Based on a Modified Grey Wolf Optimizer. Sensors, 20(3):820-820.

36. Zou, H. (2020). Clustering Algorithm and Its Application in Data Mining. Wireless Personal Communcation, 110:21-30. 